\title{
Immunostaining for CD31 and CD34 in Kaposi sarcoma
}

\author{
R Russell Jones, G Orchard, B Zelger, E Wilson Jones
}

\begin{abstract}
Aims-To evaluate antibodies directed against CD31 (JC70/A) and CD34 (QBEND/10 and anti-HPCA-1) more extensively in Kaposi sarcoma; to assess their value in routine diagnosis; and to compare them with the traditional endothelial cell markers Ulex europaeus agglutinin 1 (UEA-1) and factor VIII related antigen. Methods-Twenty four cases of Kaposi sarcoma were studied retrospectively. All specimens had been fixed in formalin and embedded in paraffin wax. The antibodies were applied using the Streptavidin biotin technique in all cases except for UEA-1, for which an indirect two stage method was used involving peroxidase conjugated anti-ulex as the secondary antibody.

Results-Tumours were classified into those showing angiomatoid or lymphangiomatoid elements and spindle cell lesions. Universal labelling of all lesions and virtually all elements within lesions was seen with the anti-CD34 antibodies QBEND/10 and HPCA-1. Labelling of spindle cells was less consistent with JC70/A but both markers were superior to the traditional endothelial cell markers UEA-1 and factor VIII related antigen. Conclusions-These data confirm that Kaposi sarcoma is a tumour of endothelial cell origin. They shed further light on the histogenesis of this complex tumour and demonstrate that immunostaining for CD34 and CD31 can be used as an aid to diagnosis in routinely processed tissue. (f Clin Pathol 1995;48:1011-1016)
\end{abstract}

Keywords: Kaposi sarcoma, CD31, CD34.

Immunohistochemistry has been used extensively in the study of malignant vascular tumours; firstly, to confirm their endothelial cell origin and, secondly, to aid diagnosis in routine histopathology. However, many of the markers which have been used for research purposes cannot be applied to routinely processed tissue because the antigenic determinants do not survive formalin fixation or embedding in paraffin wax. Thus our own studies using monoclonal antibodies such as PAL-E and EN4, ${ }^{12}$ while valuable in confirming the endothelial cell nature of Kaposi sarcoma and other malignant vascular tumours, cannot be used for evaluating routinely processed specimens. These restrictions do not apply to the traditional endothelial markers, Ulex europaeus agglutinin 1 (UEA-1) and factor VIII related antigen. However, studies of Kaposi sarcoma and angiosarcoma have produced conflicting results, particularly in relation to factor VIII related antigen. ${ }^{34}$

Monoclonal antibodies directed against CD34 and CD31 have excited interest in the study of vascular tumours. While they are not endothelial cell specific, they are widely expressed in vascular endothelium, particularly in pathological states. There is also some doubt whether they will label lymphatic endothelium. ${ }^{5-8}$ Only limited studies of Kaposi sarcoma have been carried out using antibodies directed against CD34 or CD31 and some of these have used cryostat sections. Our study aimed to evaluate these markers more extensively in Kaposi sarcoma, to assess their value in routine diagnosis and to compare them with the traditional endothelial cell markers UEA-1 and factor VIII related antigen.

\section{Methods}

Twenty four cases of Kaposi sarcoma were retrieved from the files of St John's Dermatopathology department, St Thomas's Hospital, London, and the Department of Dermatology, University of Innsbruck, Austria. All specimens had been fixed in formalin and embedded in paraffin wax. The endothelial cell markers included UEA-1 (Sigma, Poole, Dorset, UK; diluted 1 in 50) and four monoclonal antibodies: anti-factor VIII related antigen (Dako, High Wycombe, UK; diluted 1 in 40); QBEND/10 (Oxoid, Basingstoke, UK; diluted 1 in 50), JC70/A (Dako; diluted 1 in 10); and anti-HPCA-1 (Becton Dickinson, Gosport, UK; diluted 1 in 25). The Streptavidin biotin technique was used in all cases except for UEA-1, for which an indirect two stage method was used involving peroxidase conjugated anti-ulex as the secondary antibody. All tissue sections were blocked for endogenous peroxidase for 10 minutes prior to immunolabelling using a 3\% hydrogen peroxide in methanol solution. Sections for factor VIII related antigen and UEA-1 labelling were trypsinised in the normal manner for 15 minutes (Sigma). JC70/A labelling required protease digestion (Sigma Type $\mathrm{X}$ bacillus thermoproteolyticus rokko) for 30 minutes. QBEND/ 10 and HPCA-1 did not require predigestion.

\section{Results}

\section{HISTOLOGY}

Traditionally, Kaposi sarcomas are divided into patch, plaque or nodular lesions. In our study 13 biopsy specimens were patch or plaque 

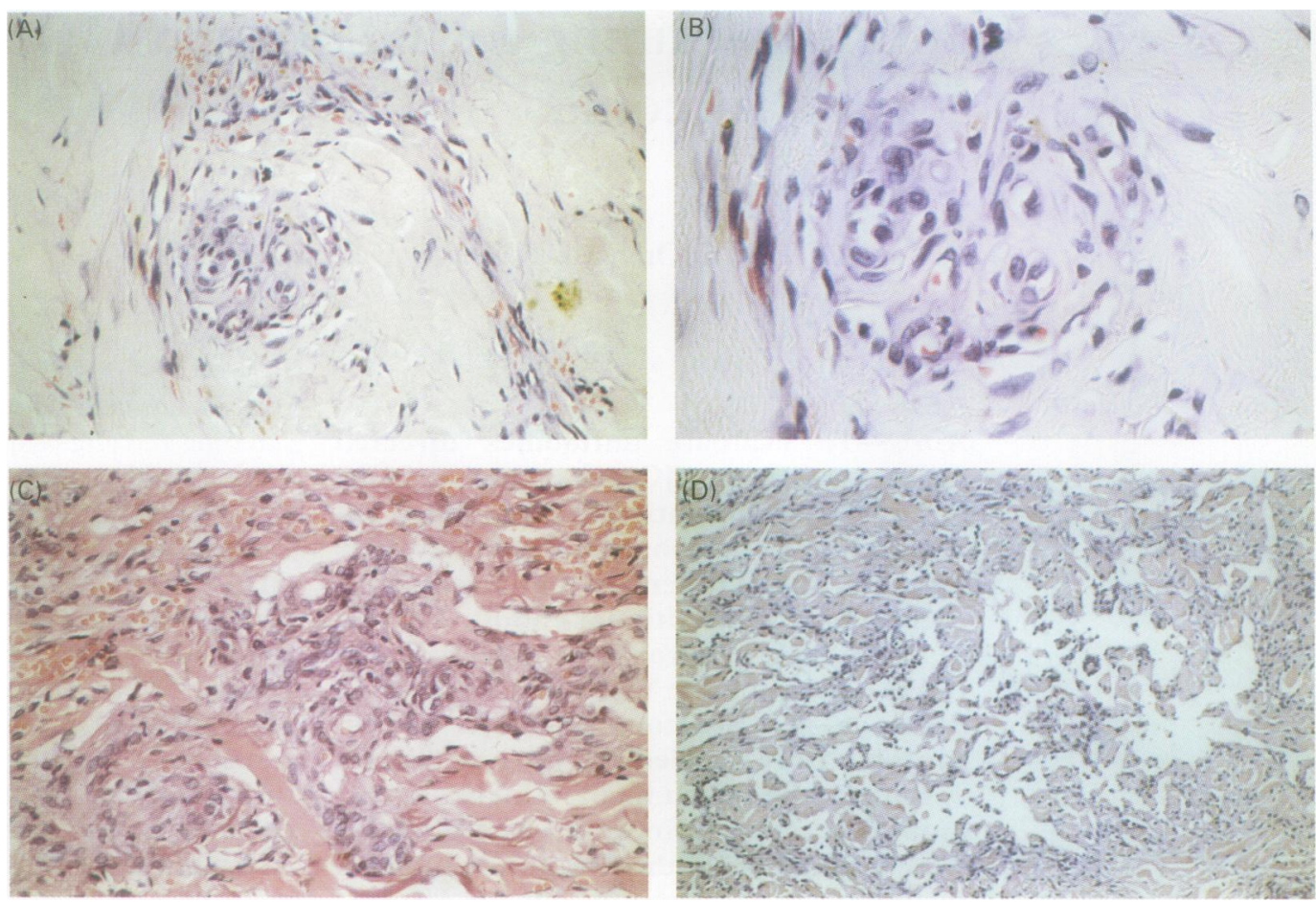

Figure 1 (A) Patch stage lesion showing angiomatoid foci with early spindle cell formation (original magnification, $\times 10$ ). (B) High power of angiomatoid focus (original magnification, $\times 40$ ). (C) Plaque stage lesion showing a mixed pattern consisting of angiomatoid foci and lymphatic slits in the surrounding connective tissue (original magnification, $\times 25$ ). (D) Plaque stage lesion showing lymphangiomatoid changes which have merged to produce an angiosarcoma-like appearance (original magnification, $\times 10$ ).

lesions, and 11 were nodular. However, from a histogenetic point of view it is more informative to concentrate on the different elements within a Kaposi lesion, particularly during the earliest phases of development. At this stage two types of lesion can be seen histologically. Angiomatoid lesions are small foci of vascular proliferation associated with a few connective tissues cells, a variable inflammatory cell infiltrate, and some red cell extravasation. The angiomatoid foci usually contain a central vessel with prominent endothelial cells surrounded by one or two thinwalled vessels containing erythrocytes and resembling small capillaries (fig $1 \mathrm{~A}$ and $1 \mathrm{~B}$ ). The lumina of these small vessels can become dilatated so that adjacent lesions are separated only by a thin endothelial cell lining, the socalled "back to back" capillaries.
The second type of lesion consists of thinwalled, bloodless vessels dispersed within the connective tissue. These vessels are angulated rather than rounded and resemble small lymphatics. This so called lymphangiomatoid variant is seen commonly, but not exclusively, in HIV related diseases. Occasionally, the lymphaticlike vessels merge to produce a dissection of collagen, similar in appearance to that seen in well differentiated angiosarcomas (fig 1D). ${ }^{9}$

Both lymphangiomatoid and angiomatoid changes may be seen within the same lesion (fig 1C). Of the $13 \mathrm{patch} / \mathrm{plaque}$ stage lesions studied, two showed lymphangiomatoid changes only, four showed angiomatoid changes only, and seven showed a mixed pattern. Areas of spindle cell proliferation are sometimes seen in these early lesions, particularly in relation to angiomatoid foci (fig 1A).

Immunocytochemical labelling profile of five monoclonal antibodies in 24 cases of Kaposi sarcoma

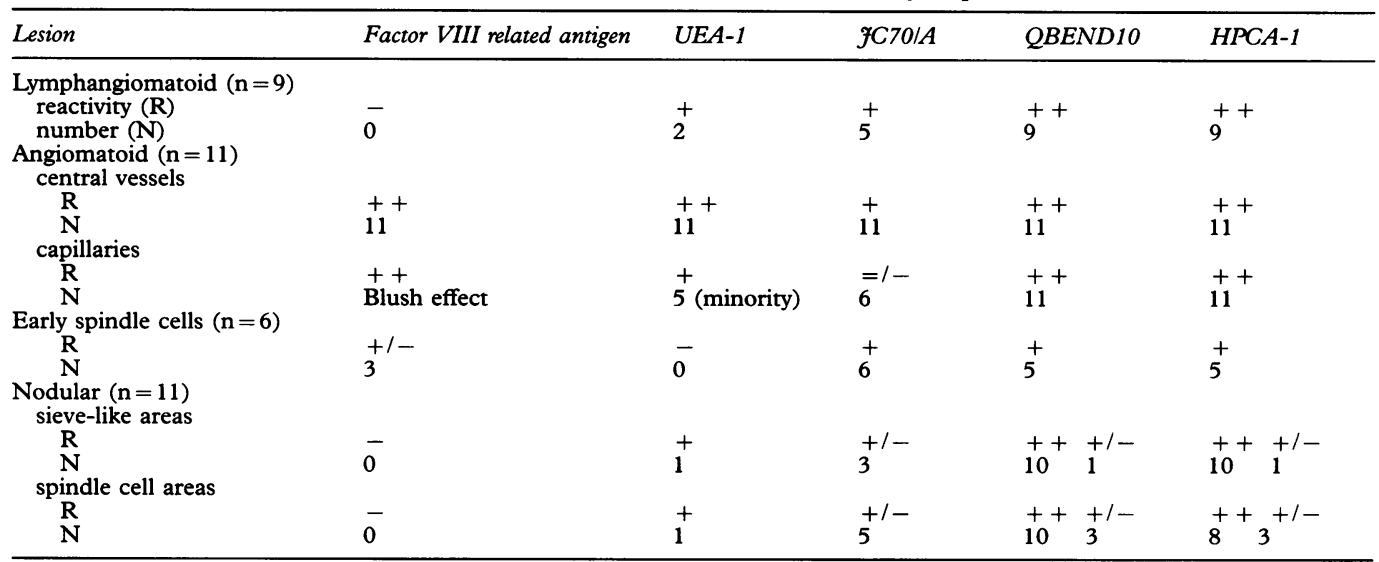



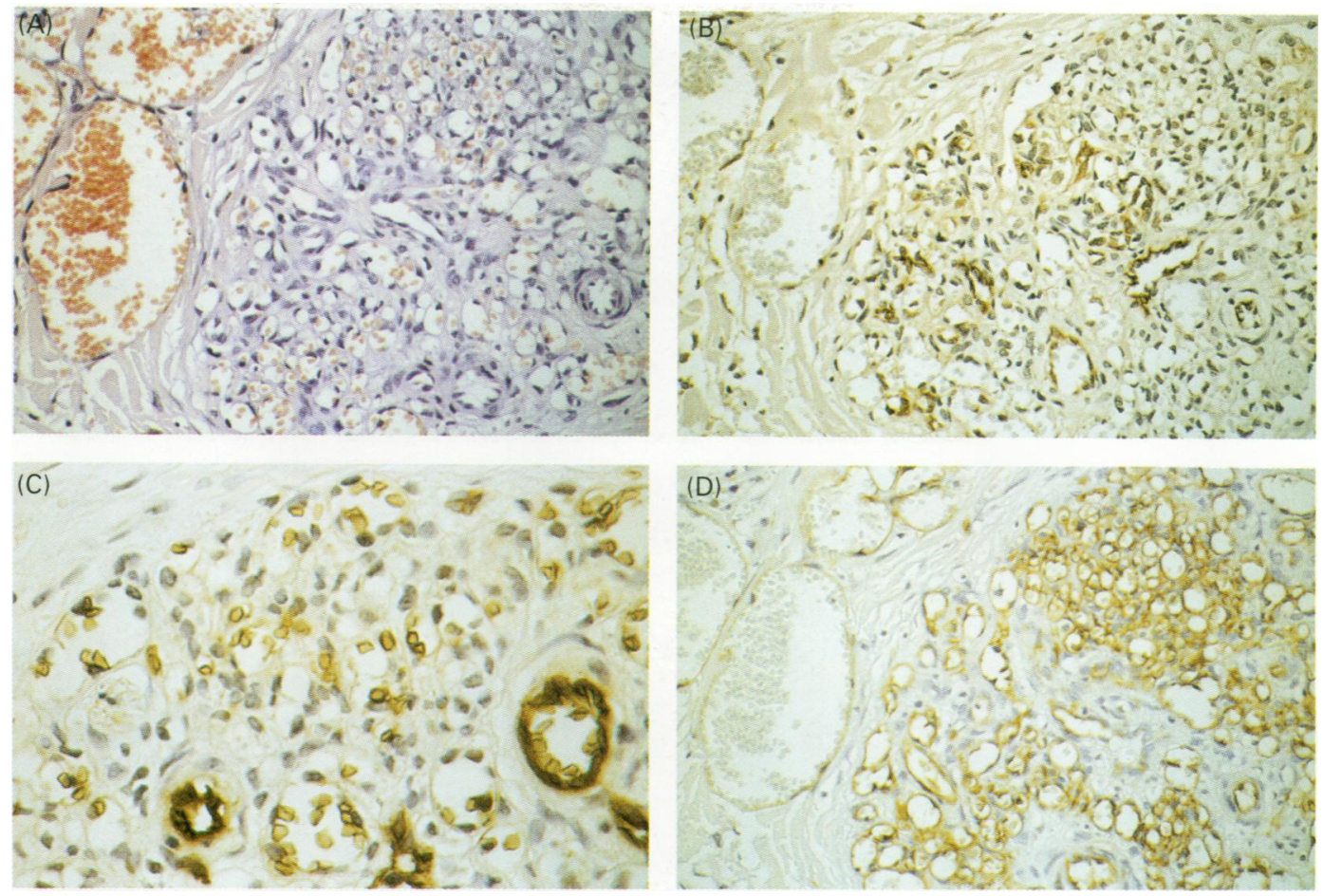

Figure 2 (A) Sieve-like areas with adjacent large back to back capillaries (original magnification, $\times 10$ ). (B) $\mathcal{F} C 70 / A$ (CD31) labels well differentiated vessels, but the smaller capillary-like vessels and the back to back capillaries are negative (original magnification, $\times 10$ ). (C) UEA-1 shows a similar pattern of labelling to $7 C 70 / A$. Capillaries are negative but contain positively stained red blood cells, which can give an appearance of false positivity (original magnification, $\times 40$ ). (D) Equivalent area to $(A)$ and $(B) . Q B E N D / 10$ shows positive labelling of all vessels and capillary-like channels within the sieve-like area. The dilatated back to back capillaries are only weakly positive (original magnification, $\times 10$ ).

In this study six of the 13 early lesions showed spindle cell changes. Eventually, however, the different foci expand and merge to form a
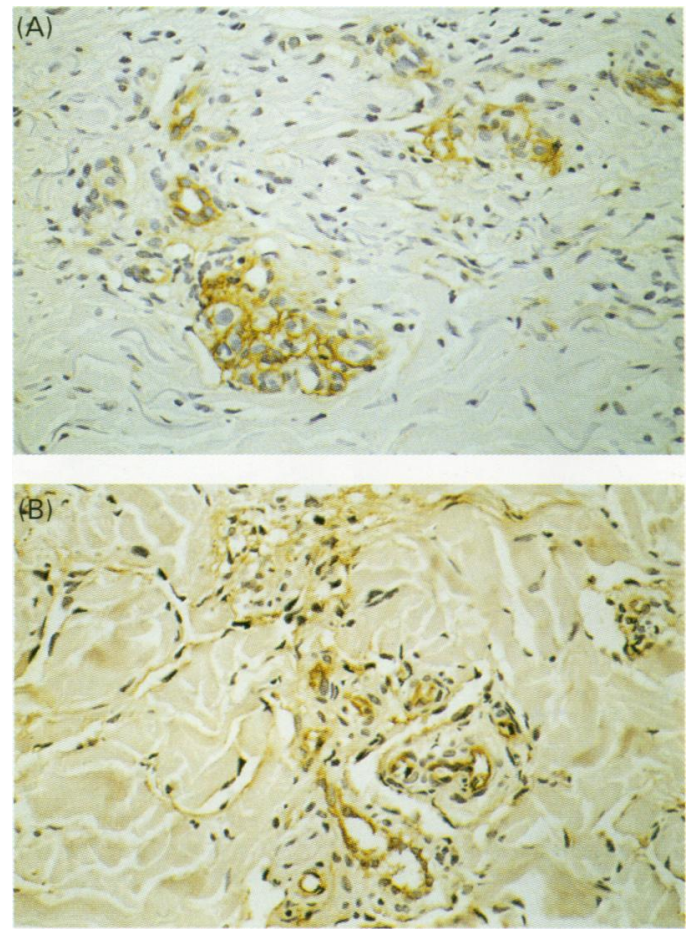

Figure 3 Angiomatoid focus with surrounding slit-like spaces (mixed pattern). (A) Diffuse factor VIII related antigen labelling of angiomatoid focus. The lymphatic-like slits are negative (original magnification, $\times 20$ ). (B) fC70/A (CD31) showing positive labelling of well differentiated central vessel and adjacent capillaries within the angiomatoid focus. The surrounding lymphatic slits are also weakly positive (original magnification, $\times 20$ ). network of tissue containing a mixture of vascular and spindle cell elements. In some areas numerous small capillaries are cut in crosssection to give an appearance which has been likened to a sieve (fig $2 \mathrm{~A}$ ). In other areas sheets of spindle cells separated by slit-like spaces containing erythrocytes are evident. These are the histological features classically associated with nodular lesions of Kaposi sarcoma. In later lesions spindle cells may predominate with sieve-like areas absent or confined to the periphery of the nodule.

\section{IMMUNOCYTOCHEMISTRY}

The staining profile seen with the different elements in the 24 lesions of Kaposi sarcoma studied is summarised in the table. Figures 2 to 5 illustrate the important findings.

Factor VIII related antigen was consistently positive only in the angiomatoid foci. In addition to staining of the central vessels and surrounding capillaries, there was also diffusion of factor VIII related antigen leading to a blush effect and non-specific staining of the surrounding connective tissue cells (fig 3A). Similar foci were seen within nodular lesions, but factor VIII related antigen did not stain lymphangiomatoid lesions, sieve-like areas, or spindle cells (fig 4A).

Staining with UEA-1 was also largely negative. Apart from central vessels within angiomatoid foci there were no elements consistently stained by this marker. Only one nodular lesion showed positivity in sieve-like and spindle cell areas. However, staining of red cells within nodular lesions often gave a false 

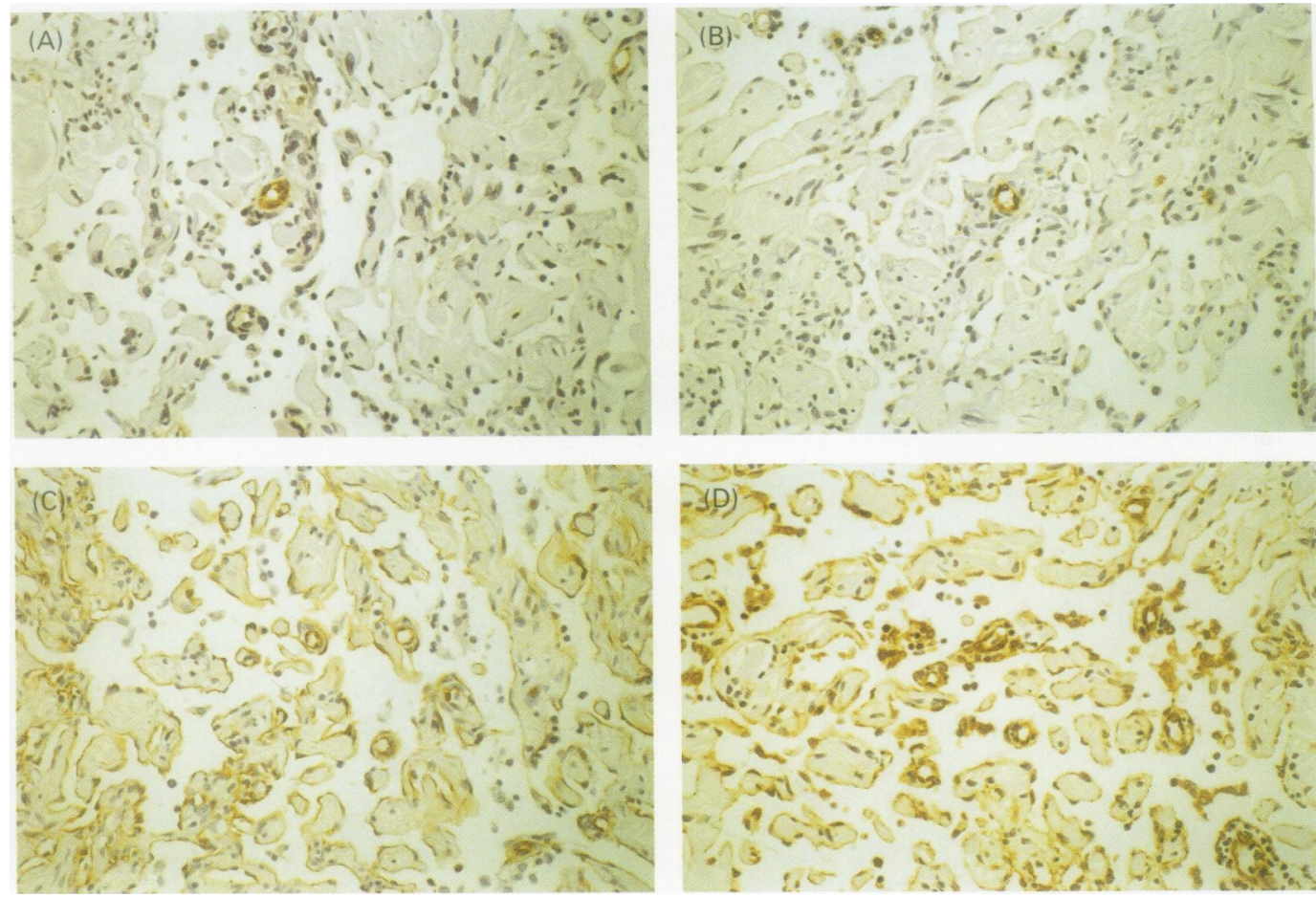

Figure 4 Pseudo-angiosarcoma lesion. (A) Factor VIII related antigen negative with positive normal central vessel (original magnification, $\times 25$ ). (B) UEA-1 negative with positive normal central vessel (original magnification, $\times 20$ ) (C) QBEND/10 positive for all cells lining the lymphangiomatoid channels (original magnification, $\times 25$ ).

(D) $\mathscr{F} C 70 / A$ (CD31) positive for all lining cells and some intraluminal cells (original magnification, $\times 25$ ).
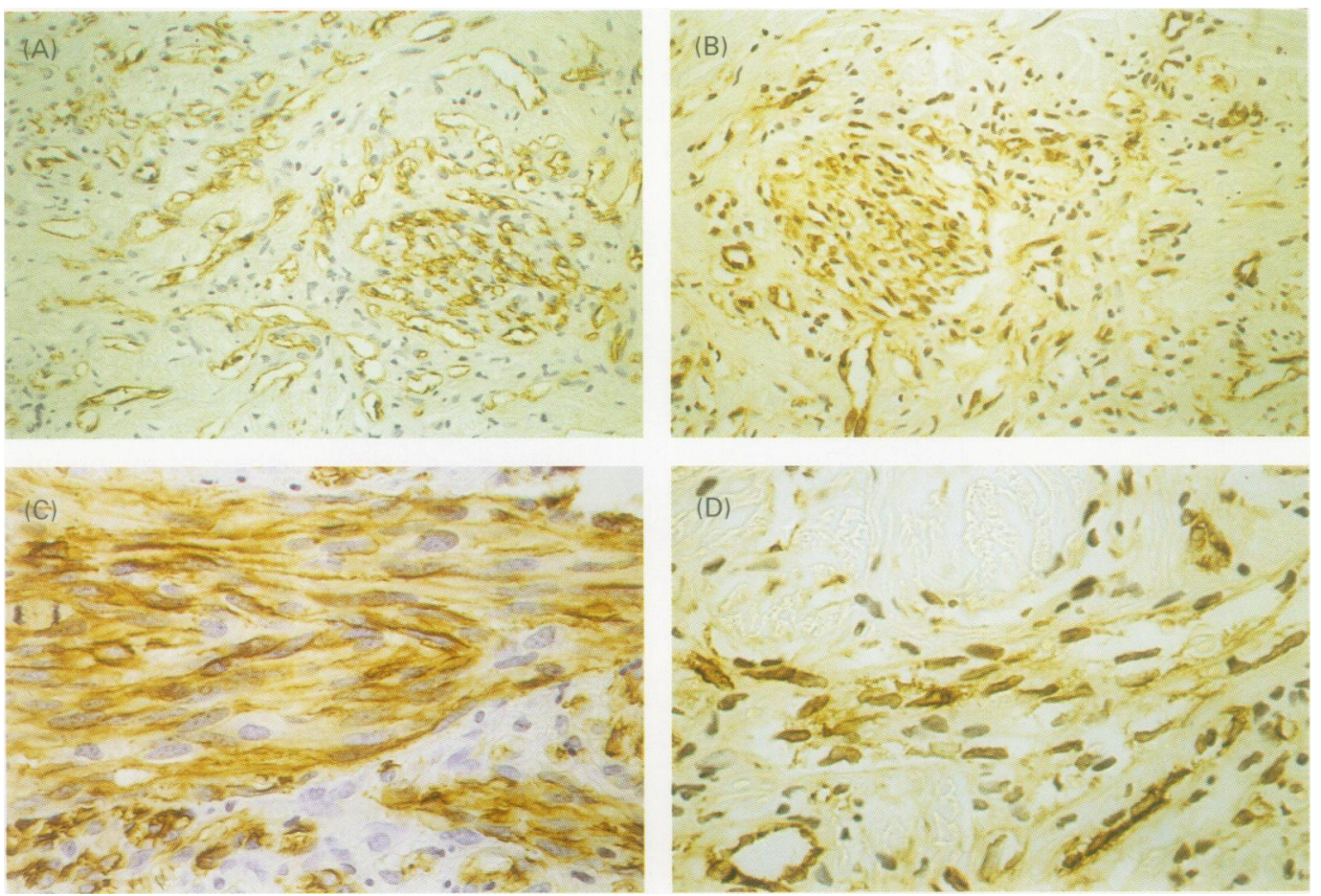

Figure 5 Spindle cell changes. (A) QBEND/10. Early spindle cell formation. The cells lining the slit-like spaces in an early spindle cell formation are particularly well demonstrated (original magnification, $\times 20$ ). (B) Equivalent area to (A). $\Upsilon_{C 70 / A}$. The spindle cells are positive but the labelling is granular and cytoplasmic rather than membranous. The slit-like spaces are poorly defined (original magnification, $\times 20$ ). (C) Nodular lesion of Kaposi sarcoma. Spindle cells are positive with HPCA-1 (original magnification, $\times 40$ ). (D) Early spindle cell formation, positive with $¥ C 70 / A-n o t e$ granular cytoplasmic labelling (original magnification, $\times 40$ ).

appearance of positivity (fig 2C). A few capillaries in angiomatoid foci and in two of nine lymphangiomatoid lesions stained with UEA1 (fig 4B).

By contrast, the CD34 antibodies provided exceptionally consistent labelling of all ele- ments within the lesions of Kaposi sarcoma. The luminal surfaces of all vascular elements were particularly clearly delineated by these markers which often permitted the appreciation of vessels not apparent on haematoxylin and eosin stained sections. There were no sig- 
nificant differences between the staining profiles for the two CD34 antibodies, QBEND/ 10 and HPCA-1. All lymphangiomatoid lesions were labelled (fig 4C) as well as all vessels and capillaries within angiomatoid lesions. Connective tissue cells in early angiomatoid lesions were not labelled but all lesions showing early spindle cell formation except one were positive for CD34 (fig 5A). In nodular lesions the sievelike areas were particularly well visualised with only one tumour showing weak staining in these areas (fig 2D). CD34 also labelled spindle cells (fig 5C) but in three predominantly spindle cell lesions the labelling was patchy, particularly towards the centre of the nodule.

The CD31 antibody JC70/A was less consistent than the CD34 markers. Thus, only five of nine lymphangiomatoid lesions were labelled by this antibody (fig 5D). Staining of angiomatoid foci was limited to the central vessels with only a few capillaries showing weak labelling (fig 3B). In nodular lesions the sieve-like areas were also poorly stained by this antibody (fig 2B). However, a few of the connective tissue cells within the angiomatoid foci and all of the six lesions showing early spindle formation were stained by CD31 (fig 5B). By contrast, labelling of spindle cells within nodular lesions was weak and patchy in five cases and negative in six. CD31 labelling was cytoplasmic and granular and less easy to define than CD34 (fig 5D).

\section{Discussion}

Previous studies of Kaposi sarcoma using antibodies directed against CD31 and CD34 have been limited. Ramani et $a \hat{l}$ in a study of 40 cases reported positive CD34 labelling of lymphangiomatoid elements with variable staining of spindle cell elements. Parums et al reported negative CD31 labelling in spindle cells of four cases of Kaposi sarcoma, whereas Nickoloff reported positive labelling on cryostat sections. ${ }^{8}$ The data presented here demonstrate that CD34, and to a lesser extent CD31, antibodies are more reliable at labelling Kaposi sarcoma lesions than the traditional endothelial cell markers, UEA-1 and factor VIII related antigen, and can be used on routinely processed tissue. For Kaposi sarcoma CD34 seems to be superior to CD31. All cases of Kaposi sarcoma labelled with QBEND/10 and HPCA-1, whereas four of nine lymphangiomatoid lesions and the majority of nodular lesions were negative with JC70/A. Furthermore, the pattern of labelling for QBEND/10 was more clearly defined than the diffuse pattern seen with JC70/A.

These findings contrast with our own studies in angiosarcoma where CD31 was found to be a more reliable marker than CD $34 .{ }^{10}$ Our findings also contrast with those of Suster and Wong ${ }^{11}$ who reported essentially negative staining with HPCA-1 in 20 cases of Kaposi sarcoma. This discrepancy may be due to technical factors as we used the same antibody but at a 1 in 25 dilution compared with the 1 in 200 dilution used in the study by Suster and Wong. It has been suggested that QBEND/10 and HPCA-1 label different epitopes on CD34. ${ }^{11}$ Our findings, however, do not support this hypothesis in that similar labelling of Kaposi lesions was found with both monoclonal antibodies.

Staining with the pan endothelial cell marker UEA-1 was less reliable in Kaposi sarcoma than either CD34 or CD31. Only one of 11 nodular lesions and two of nine lymphangiomatoid lesions labelled with UEA-1. By contrast, UEA-1 is a reliable marker for angiosarcoma in formalin fixed tissue. In a previous study ${ }^{10}$ it labelled 18 of 19 cases in both formalin and PLP (periodate lysine paraformaldehyde) fixed tissue.

Our findings demonstrate that CD34 is the best marker for labelling Kaposi sarcoma lesions in routinely fixed tissue and shed some light on the histogenesis of Kaposi sarcoma. Thus, the spindle cells seen in early patch and plaque stage lesions are CD31 positive in all cases and CD34 positive in virtually all cases. By contrast, in nodular lesions of Kaposi sarcoma the majority of spindle cells are still CD34 positive but a significant number are now CD31 negative. In late lesions where spindle cells predominate, CD34 labelling also becomes more variable. This provides evidence of de-differentiation and demonstrates that loss of vascular markers is important within spindle cell lesions.

CD34 is a cell surface protein coded for by the CD34 gene on chromosome lq/32. It is expressed by human haematopoietic cells of both the myeloid and lymphoid series as well as endothelial cells. It may have a role in regulating early events in blood cell differentiation or it may function as an adhesion molecule in both endothelial cells and haematopoietic progenitor cells. CD34 is said to be present on normal venular but not lymphatic endothelium, and has been reported as negative in lymphangiomas. ${ }^{6}$ If so, this would strongly favour a vascular origin for the spindle cell elements in Kaposi sarcoma. However, Ramani et a $\bar{l}$ have reported that five of eight cases of lymphangioma were focally positive with QBEND/ 10 and it is possible that adhesion molecules are expressed more strongly in pathological states. In our own studies some sections showed positive labelling of lymphangiomatoid elements with negative labelling of adjacent "normal" lymphatics. Similar considerations apply to CD31. Again, CD31 is thought to label vascular rather than lymphatic endothelium and is reportedly absent in lymphangiomas and lymphoepithelial cysts. ${ }^{7}$ However, Nickoloff found CD31 labelling on both vascular and lymphatic epithelium using cryostat sections, ${ }^{8}$ and in the present study some normal lymphatics (that is, those containing small valves) labelled with CD31.

It would seem therefore that in Kaposi sarcoma CD34 and CD31 markers do not help to distinguish between cells derived from lymphatic or vascular endothelium. Rather it suggests that the immunocytochemical profile of the endothelial derived cells in Kaposi sarcoma are unlike those found in normal tissue. Rather than interpreting the immunocytochemical findings in terms of "derivation from", it might be more appropriate to regard 
the endothelial cells of Kaposi sarcoma as pathological tissue which has differentiated away from normal endothelium. Certain elements such as the angiomatoid foci still resemble vascular tissue, but by the time spindle cells have come to predominate it is no longer possible to relate endothelial cells to their normal counterparts. In addition, the multifocal nature of Kaposi sarcoma indicates that more than one type of endothelial cell could be involved in its histogenesis.

1 Russell Jones R, Spaull J, Spry C, Wilson Jones E. Histogenesis of Kaposi's sarcoma in patients with and without acquired immune deficiency syndrome (AIDS) $\mathcal{F} \mathrm{Clin} \mathrm{Pa}-$ thol 1986;39:742-9.

2 Holden C, Spaull J, Williams R, Spry C, Russell Jones R Wilson Jones $\mathrm{E}$. The detection of endothelial cell antigens in cutaneous tissue using methocam and periodate lysine in cutaneous tissue using methocam and periodate lysine paraformal

3 Leader M, Collins M, Patel J, Henry K. Staining for factor VIII related antigen and Ulex europaeus agglutinin 1 (UEA1) in 230 tumours. An assessment of their specificity fo angiosarcoma and Kaposi's sarcoma. Histopathology 1986 10:1153-62.
4 Burgdorf W, Muhai K, Rosai J. Immunohistochemical identification of factor VIII-related antigen in endothelial cells of cutaneous lesions of alleged vascular nature. $\mathrm{Am} \mathcal{7} \mathrm{Clin}$ Pathol 1981;75:169-71.

5 Ramani P, Bradley NJ, Fletcher CDM. QBEND/10, a new monoclonal antibody to endothelium: assessment of its diagnostic utility in paraffin sections. Histopathology 1990 17:237-42.

6 Sankey E, More L, Dhillon A. QBEND/10: A new immunostain for the routine diagnosis of Kaposi's sarcoma munostain for the routine

7 Parums DV, Cordell JL, Micklem K, Heryet AR, Gatter KC, Mason DY. JC70. A new monoclonal antibody that detects vascular endothelium associated antigen on routinely processed tissue sections. F Clin Pathol 1990;43 $752-7$.

8 Nickoloff B. PECAM-1 (CD31) expressed as proliferating endothelial cells, stromal spindle-shaped cells and derma dendrocytes in Kaposi's sarcoma. Arch Dermatol 1993;129. 250-1.

9 Gange RW, Wilson Jones E. Lymphangioma-like Kaposi's sarcoma. Br f Dermatol 1979;100:327-34.

10 Orchard GE, Zelger B, Wilson Jones E, Russell Jones R. An immunohistochemical assessment of 19 cases of cutaneous immunohistochemical assessment of 19 cases of

11 Suster S, Wong T-Y. On the discriminatory value of antiSuster S, Wong T-Y. On the discriminatory value of anti-
HPLA-1 (CD34) is the differential diagnosis of benign HPLA-1 (CD 34) is the differential diagnosis of benign Dermatopathol 1994;16:355-63.

12 Svanholm H, Neilsan K, Horge P. Factor VIII related antigen and lymphatic collecting vessels. Virchows Arch $A$ Pathol Anat Histopathol 1984;404:223-8. 